\title{
Application of Lie Symmetry Analysis to Heat and Mass Transfer Equations ${ }^{\dagger}$
}

\author{
Irina Stepanova \\ Institute of Computational Modelling of SB RAS, 660036 Krasnoyarsk, Akademgorodok, Russia; \\ stepiv@icm.krasn.ru \\ + Presented at Symmetry 2017-The First International Conference on Symmetry, Barcelona, Spain, \\ 16-18 October 2017.
}

Published: 3 January 2018

This talk is devoted to the study of equations describing the thermal diffusion process in a binary mixture via the Lie symmetry approach. Thermal diffusion is the component separation in nonuniformly heated fluid. The mathematical model of the process is described by Navier-Stokes equations and mass and heat transport equations.

The group classification problem for thermal diffusion equations is solved in correspondence with five arbitrary functions concerning the physical properties of the mixture. Some invariant solutions of the governing equations are constructed and applied to describe the thermal diffusion convection in the simple physical applications.

Special attention is paid to the thermal diffusion equations without convection. We take into account the thermal diffusion conductivity as well as simple thermal diffusion. The symmetries of these equations are found based on the forms of the transport coefficients. Some theorems concerning the general symmetry properties of such equations are proven.

Acknowledgments: This work is supported by the Russian President Grant (project MK-4519.2016.1).

Conflicts of Interest: The authors declare no conflict of interest.

(C) 2018 by the author. Licensee MDPI, Basel, Switzerland. This article is an open access article distributed under the terms and conditions of the Creative Commons Attribution (CC BY) license (http://creativecommons.org/licenses/by/4.0/). 